\title{
Severe adverse events during second-line tuberculosis treatment in the context of high HIV Co-infection in South Africa: a retrospective cohort study
}

Kathryn Schnippel ${ }^{1,2,3^{*}}$ (D), Rebecca H. Berhanu ${ }^{1,4,5}$, Andrew Black ${ }^{6}$, Cynthia Firnhaber ${ }^{1,2}$, Norah Maitisa ${ }^{6,7}$, Denise Evans ${ }^{4}$ and Edina Sinanovic ${ }^{3}$

\begin{abstract}
Background: According to the World Health Organization, South Africa ranks as one of the highest burden of TB, TB/HIV co-infection, and drug-resistant TB (DR-TB) countries. DR-TB treatment is complicated to administer and relies on the use of multiple toxic drugs, with potential for severe adverse drug reactions. We report the occurrence of adverse events (AEs) during a standardised DR-TB treatment regimen at two outpatient, decentralized, public-sector sites in Johannesburg, South Africa.

Methods: We reviewed medical records of the six-month intensive treatment phase for rifampicin-resistant (RR) TB patients registered May 2012 - December 2014. Patients contributed follow-up time until death, loss from treatment, censoring (6 months) or data extraction. A standardized regimen of kanamycin, moxifloxacin, ethionamide, terizidone, and pyrazinamide was used according to national guidelines. AEs were graded using the AIDS Clinical Trial Group scale. We present subhazard ratios from competing risk analysis for time to severe $\mathrm{AE}$, accounting for mortality and loss from treatment.

Results: Across the two sites, 578 eligible patient files were reviewed. $36.7 \%$ were categorized as low weight ( $\leq 50 \mathrm{~kg}$ ) at DR-TB initiation. $76.0 \%$ had no history of TB treatment prior to the current episode of RR TB. $26.8 \%$ were diagnosed with RR TB while hospitalized, indicating poor clinical condition. $82.5 \%$ of patients were also HIV positive, of whom 43. $8 \%$ were on ART prior to RR TB treatment and $32.1 \%$ initiated ART with or after RR TB treatment. Median CD4 count was 114.5 (IQR: 45-246.5). Overall, 578 reports of AEs were captured for 204 patients (35.3\%) and 110 patients (19.0\%) had at least one severe AE reported. Patients with at least one AE experienced a median of 3 (IQR: 2-4) AEs per patient. HIV-positive patients with CD4 counts $\leq 100 \mathrm{cell} / \mathrm{s} / \mathrm{mm}^{3}$ and those newly initiating ART were more likely to experience a severe AE (sHR: 2.76, 95 \% Cl: 1.30-5.84 and sHR: 3.07, 95 \% Cl: 1.46-6.46, respectively).
\end{abstract}

Conclusion: Severe AE are common during the first 6 months of RR TB treatment and HIV-positive patients newly initiating ART have the highest subdistribution hazard ratio for severe $A E$, accounting for the competing risks of death and loss from treatment.

Keywords: HIV, Antiretroviral therapy, Multi-drug resistant TB, Tuberculosis, Adverse drug reactions

\footnotetext{
* Correspondence: kschnipp@gmail.com

${ }^{1}$ Right to Care, Johannesburg, South Africa

${ }^{2}$ Clinical HIV Research Unit, School of Clinical Medicine, Faculty of Health

Sciences, University of the Witwatersrand, Johannesburg, South Africa

Full list of author information is available at the end of the article
} 


\section{Background}

South Africa has made progress in controlling the TB epidemic; the 2015 Global Tuberculosis Report highlights a declining incidence and prevalence of $\mathrm{TB}$ for South Africa [1]. Despite the progress seen tuberculosis has been the most common cause of death in South Africa from 2005 to 2014 [2] and the number of persons diagnosed with drug-resistant (DR-TB) TB has increased significantly over the last decade from 2000 patients in 2005 to 18,734 in 2014 [1].

In 2011, South Africa adopted a policy of universal rifampicin (RIF) resistance testing using Xpert MTB/RIF (Cepheid, USA) as the first-line TB diagnostic in the country. Reporting of DR-TB now focuses on RIF resistant TB (RR TB), which includes RIF resistance with unknown or pending sensitivities to other drugs, mono-RIF resistant $\mathrm{TB}$, multi-drug resistant (MDR) $\mathrm{TB}$ that is resistant to both RIF and isoniazid (INH), extensively drug resistant (XDR) TB which is MDR TB plus resistance to second-line drugs from the fluoroquinolone and injectable aminoglycoside or cyclic peptide classes, or preXDR TB which is MDR TB plus resistance to either a fluoroquinolone or a second-line injectable drug [3]. The South African National TB Programme (NTP) treatment guidelines for RR-TB indicate 18-24 months of treatment [4]. All RR TB patients are started on the standardized second-line MDR TB regimen until further resistance is either confirmed or ruled-out at which point the patient may be switched to an individualized second-line TB regimen (if preXDR or XDR TB) or INH may be added to the regimen (if mono-RIF resistant) [4].

Second-line TB treatment is complicated to administer, with frequent and potentially severe adverse drug reactions (ADR) $[5,6]$. Adding to the complexity of treatment, an estimated $60 \%$ of all TB patients [1] and up to $80 \%$ of RR-TB patients in South Africa [7] are also HIVinfected and therefore eligible for antiretroviral therapy (ART). RR TB treatment and ART present overlapping toxicities which may be worsened by concomitant use, for example both kanamycin and tenofovir may cause renal dysfunction [8].

ADR can negatively impact the effectiveness of RR TB treatment in many ways. Patients or clinicians may interrupt, reduce dosage, or stop treatment in an attempt to alleviate side effects. This results in an increased risk of acquiring additional drug resistance, failing treatment, or dying from TB. ADR themselves may also result in hospitalization, permanent disability, or death. Only $49 \%$ of the 2012 cohort of MDR TB patients in South Africa were cured or successfully completed treatment, below both the global average (50\%) and targets (75\%) [1]. Thus, evidence of the burden and risks of ADR during RR TB treatment is important for both patients and clinicians to manage this complexity. In order to quantify the burden of ADR during outpatient RR TB treatment in the context of high co-infection with HIV, we present the results of a medical file review of routinely reported adverse events (AE) from two decentralised public-sector sites within South Africa.

\section{Methods}

The study was conducted at the TB Focal Point clinics at Helen Joseph and Charlotte Maxeke Academic Hospitals in Johannesburg, South Africa. Patients with laboratory diagnosis of pulmonary or extrapulmonary RR TB from any level of health facility in the catchment area are referred to these NGO-supported, outpatient, public-sector facilities for initiation of second-line TB treatment and further drug resistance testing. Patients are referred from surrounding primary health care centres, private facilities, Helen Joseph and Charlotte Maxeke inpatient wards, and surrounding hospitals. A census of medical files for patients who enrolled for second-line TB treatment at Helen Joseph (May 2012 to June 2014) or Charlotte Maxeke (May 2012 to December 2014) were reviewed retrospectively in September 2014 and April 2015, respectively. Only patients with documentation of at least rifampicin resistance, i.e. RIF resistance detected by Xpert, RIF monoresistance, MDR-TB diagnosed by LPA, or XDR TB were included in the study.

\section{Standard of care treatment}

Upon referral the patient undergoes HIV counselling and testing, patients are educated about the length and toxicities of RR TB treatment, and informed about the importance of screening exposed family members. Patients are then examined and evaluated by a medical officer. All RIF resistance diagnosed by Xpert is treated presumptively as MDR TB as per the South African National TB Programme (NTP) guidelines [4]. The intensive phase of treatment (approximately 6 months) consists of a five-drug regimen including the second-line injectable kanamycin, moxifloxacin, ethionamide, terizidone, and pyrazinamide $[4,9]$. The continuation phase of treatment (18 to 24 months) includes moxifloxacin, ethionamide, terizidone, and pyrazinamide. Treatment dose is adjusted for patient weight, with patients 33 to $50 \mathrm{~kg}$ receiving smaller doses of kanamycin $(500-750 \mathrm{mg}$ vs. $1000 \mathrm{mg}$ ), ethionamide ( $500 \mathrm{mg}$ vs. $750 \mathrm{mg}$ ), and pyrazinamide (1000-1750 mg vs. $2000-2500 \mathrm{mg}$ ) than patients weighing 51 to $70 \mathrm{~kg}$ [4]. Linezolid and bedaquiline were not available at the study sites during the study period [10].

The 2010 South African ART guidelines indicated eligibility for HIV-infected patients with TB co-infection at $\mathrm{CD} 4 \leq 350$ cells $\mathrm{mm}^{3}$ and for any patient with $\mathrm{CD} 4 \leq 200$ cells $\mathrm{mm}^{3}$ [11]. Eligibility was expanded in 2013 to include all patients with CD4 count $\leq 350$ cells $\mathrm{mm}^{3}$ [12]. Thus, all 
RR TB patients not already on ART are initiated at the RR TB site. Patients currently on ART can receive ART from the RR TB site for the duration of second-line TB treatment. From 2010, most South African ART patients in the public sector are initiated on a standard, first-line three-drug regimen of tenofovir, efavirenz or nevirapine, and lamivudine or emtricitabine. From 2013, a fixed-dose combination of tenofovir, efavirenz, and emtricitabine has been the preferred first-line ART regimen [12].

Patients are asked to return at two weeks and subsequently reviewed monthly by a TB nurse or medical officer for adherence to therapy, evaluation of side effects and treatment response. Sputum samples for smear microscopy and TB culture are collected monthly in pulmonary TB patients to assess for sputum smear and culture conversion. As per NTP treatment guidelines, baseline laboratory tests are conducted prior to treatment initiation to assess for anaemia and renal, thyroid and liver function. If HIV co-infected, laboratory tests to monitor HIV treatment (CD4 count and HIV viral load) or ART toxicities are integrated. Every two months during the six-month intensive phase of treatment blood work is repeated. Additional or more frequent laboratory testing may be ordered if clinically indicated. Audiology testing for hearing loss is conducted monthly during the six month intensive phase due to the high rates of aminoglycoside induced hearing dysfunction [13]. Electrocardiograms for monitoring were not included in the guidelines for the standard RR TB regimen at the time of the study.

\section{Adverse events and drug reactions}

For most of the period under review, pharmacovigilance requirements were for targeted spontaneous reporting to the national regulatory agency, the Medicines Control Council. Programmatic pharmacovigilance for HIV and $\mathrm{TB}$, including $\mathrm{RR} T B$ was in the process of being rolled out by the National Pharmacovigilance Centre [14]. According to the regulatory form which is separate from the medical file, 'advice about voluntary reporting' requests clinicians to please report all 'serious reactions and interactions with all products' to the Medicines Control Council.

Most of the AEs identified in this file review were found in the clinical progress notes section of the medical files. Causality was not routinely documented. AEs such as weight loss could be related to adverse drug reactions from second-line TB treatment or ART, or HIV or TB morbidity. Pre-existing conditions at the time of RR TB treatment initiation were noted under patient medical history and co-morbidities and therefore not included in the adverse events. However, if pre-existing conditions worsened during treatment it would be reported.
AEs of interest were those most likely to be adverse drug reactions and defined prior to the review as including renal dysfunction, hypokalaemia, ototoxicity, vestibular dysfunction, severe anaemia, psychosis and depression, peripheral neuropathy, seizures, hypothyroidism, nausea and/or vomiting, and joint pain. Other AEs identified during the review were recorded and categorized during data analysis. Standard of care does not include routine screening for gastro-intestinal disorders such as nausea or vomiting or mental health such as depression, or insomnia; AEs captured were as reported by patients during routine visits.

AEs were graded either at the time of the event (in the clinical notes) or during the file review by the treating clinician as mild (grade 1), moderate (grade 2), severe (grade 3), potentially life-threatening (grade 4), or fatal (grade 5) according to the AIDS Clinical Trials Group grading system [15]. AEs included those detected clinically and through laboratory testing. Where outcome of the AE (e.g. death, hospitalization, permanent disability, drug discontinuation, and/or drug dose reduction) was available in the file it was also captured. Deaths that were not reported as an outcome of an $\mathrm{AE}$, i.e. those thought to be from $\mathrm{TB}$, were not re-categorised as an $\mathrm{AE}$.

\section{Statistical analysis}

We present descriptive analysis of the number of AEs by patient, types of AE reported, and severity of AEs identified from the medical record review, including frequencies and proportions.

We used time to event analysis to present the mean time from second-line TB treatment initiation to (first) severe adverse event (grade 3 or higher). Follow-up is censored at 6 months after second-line TB treatment initiation, final outcome (i.e. death or loss from treatment for at least 2 months), transfer to another site, or data extraction (September 2014 for Helen Joseph and April 2015 for Charlotte Maxeke).

We used competing risk regression method from Fine and Gray [16] to determine if occurrence of a severe $\mathrm{AE}$ was associated with a priori identified patient demographic and clinical characteristics. In time-to-event analysis, an individual can either experience the event of interest or be censored. However, in real life, an individual may experience a number of negative outcomes, not only the event of interest. Occurrence of one negative outcome means that the other negative outcomes cannot occur, and only the time to failure for the earliest event is observed. The method of competing risk regression was chosen because of the high rate of mortality and loss from treatment in South African RR TB cohorts; one of these poor outcomes could occur before the severe $\mathrm{AE}$ and therefore the severe $\mathrm{AE}$ would not occur (or would occur but never be documented). Because loss from 
treatment may represent unreported mortality, both death and loss from treatment were considered to be the risks competing with report of a severe AE. Univariate (crude) subdistribution hazard ratios (sHR) with $95 \%$ confidence intervals $(\mathrm{CI})$ are presented. All analysis was completed in Stata v14 (College Station, TX).

Characteristics considered included: HIV status (HIV negative, HIV-positive on ART prior to RR TB diagnosis and initiation defined as at least 30 days prior to RR TB treatment start, HIV-positive initiated on ART at or after RR TB initiation, or HIV positive not on ART or ART unknown); age category (10-24, 25-39, 40-54, 55 years or older), and sex. We also examined TB symptoms (report of cough, fever, weight loss, or night sweats), and markers of severity of illness: low weight at treatment initiation $(\leq 50 \mathrm{~kg})$ or diagnosed with $\mathrm{RR} \mathrm{TB}$ during hospitalization for any cause. CD4 count at RR TB treatment initiation as documented in the patient file (HIV negative, HIV-positive with CD $4>100$ cells $/ \mathrm{mm}^{3}$, or HIV-positive with CD4 $\leq 100$ cells $/ \mathrm{mm}^{3}$ ) was defined as the CD4 count measured up to 6 months prior or within the first month of treatment. Information on pre-existing conditions or self-reports of prior TB treatment (none, first-line regimen, or regimen that included streptomycin) were also considered. No patients reported having been previously treated for RR TB. All patients were on standardized treatment regimens for RR TB; patients diagnosed with XDR TB were transferred out to initiate individualized treatment at another site and therefore exposure was censored.

\section{Results}

Across the two sites, 578 files were available for review and patients eligible for the study, patient characteristics are reported in Table 1. Patients presented with RR TB at a median age of 35 (IQR: 29-42 years) and $49.0 \%$ were male. One-third of the patients $(36.7 \%)$ were categorized as low weight $(\leq 50 \mathrm{~kg})$ at initiation of second-line TB treatment, the median weight at initiation was $54 \mathrm{~kg}$ (IQR: 47.9-61.5). Only $1.2 \%$ reported a history of TB treatment with an injectable drug (streptomycin) and the majority (76.0 \%) had no history of any TB treatment prior to the current episode of RR TB. Approximately one-quarter $(26.8 \%)$ of the patients were diagnosed with RR TB while hospitalized, indicating poor clinical condition.

HIV infection was the most common co-morbidity reported as $82.5 \%(n=477 / 578)$ were HIV positive (Table 2). Of those who were HIV positive, $43.8 \%$ were on ART at least 30 days prior to RR TB treatment initiation with a median 332 days on ART (IQR: 160, 991) and $31.9 \%$ initiated ART with or after RR TB treatment with a median time to ART initiation of 26 days after RR TB treatment (IQR: 14, 42). Of the $77.8 \%$ of HIVpositive patients with $\mathrm{CD} 4$ counts reported at $\mathrm{RR} T B$
Table 1 Characteristics of patients at initiation of second-line TB treatment $(n=578)$

\begin{tabular}{|c|c|c|c|}
\hline Characteristic & Description & Count & Proportion \\
\hline \multirow[t]{2}{*}{ Sex } & Male & 283 & $49.0 \%$ \\
\hline & Female & 295 & $51.0 \%$ \\
\hline \multirow[t]{4}{*}{ Age } & $10-24$ years & 56 & $9.7 \%$ \\
\hline & $25-39$ years & 338 & $58.5 \%$ \\
\hline & $40-54$ years & 163 & $28.2 \%$ \\
\hline & $55+$ years & 21 & $3.6 \%$ \\
\hline \multirow[t]{3}{*}{ Weight (kg) } & Low weight ( $\leq 50$ kg) & 195 & $36.7 \%$ \\
\hline & $>51 \mathrm{~kg}$ & 337 & $63.3 \%$ \\
\hline & Missing & 46 & $8.0 \%$ \\
\hline \multirow[t]{2}{*}{ TB foci } & Pulmonary & 541 & $93.6 \%$ \\
\hline & Extrapulmonary only & 37 & $6.4 \%$ \\
\hline \multirow[t]{4}{*}{ Prior TB } & No history of TB treatment & 439 & $76.0 \%$ \\
\hline & Prior first-line TB treatment & 119 & $20.6 \%$ \\
\hline & $\begin{array}{l}\text { Prior TB treatment with } \\
\text { streptomycin }\end{array}$ & 7 & $1.2 \%$ \\
\hline & Unknown & 13 & $2.2 \%$ \\
\hline \multirow[t]{4}{*}{ Current TB diagnosis } & $\begin{array}{l}\text { MDR-TB (INH and RIF } \\
\text { resistance) }\end{array}$ & 182 & $31.5 \%$ \\
\hline & RIF mono-resistant TB & 198 & $34.3 \%$ \\
\hline & $\begin{array}{l}\text { RIF resistant, sensitivities } \\
\text { unknown }\end{array}$ & 191 & $33.0 \%$ \\
\hline & $\begin{array}{l}\text { XDR TB (second-line } \\
\text { resistance) }\end{array}$ & 7 & $1.2 \%$ \\
\hline \multirow[t]{2}{*}{ Presenting symptoms } & Cough & 281 & $65.9 \%$ \\
\hline & $\begin{array}{l}\text { Any of cough, weight loss, } \\
\text { fever, night sweats }\end{array}$ & 453 & $78.4 \%$ \\
\hline \multirow{2}{*}{$\begin{array}{l}\text { Sputum smear } \\
\text { microscopy }\end{array}$} & Positive (scanty or higher) & 284 & $49.1 \%$ \\
\hline & Negative or unknown & 294 & $50.9 \%$ \\
\hline \multirow{3}{*}{$\begin{array}{l}\text { Level of care at TB } \\
\text { diagnosis }\end{array}$} & Outpatient, ambulatory & 406 & $70.2 \%$ \\
\hline & Inpatient, hospitalized & 155 & $26.8 \%$ \\
\hline & Missing & 17 & $2.9 \%$ \\
\hline
\end{tabular}

MDR-TB multi-drug resistant tuberculosis, RIF rifampicin, INH isoniazid, XDR TB extensively drug-resistant tuberculosis

treatment initiation, the median CD4 was 114.5 (IQR: 45-246.5). Half (50.2 \%) were on the standard first-line regimen for the public sector ART program (tenofovir, efavirenz, and lamivudine or emtricitabine). Few other co-morbidities were reported, with no one co-morbidity affecting 10 or more patients. Diabetes mellitus (1.9\%), renal insufficiency $(1.4 \%)$, and hepatitis or liver dysfunction $(1.2 \%)$ were the three most common comorbidities other than HIV. Seven women $(2.4 \%$ of women) were pregnant during RR TB treatment.

\section{Adverse events}

Overall, 578 reports of AEs were captured for 204 patients (35.3\%) and 110 patients $(19.0 \%)$ had at least one 
Table 2 Co-morbidities, clinical conditions and chronic medications $(n=578)$

\begin{tabular}{|c|c|c|c|}
\hline Characteristic & Description & Count & Proportion \\
\hline \multirow[t]{3}{*}{ HIV status } & Negative & 95 & $16.4 \%$ \\
\hline & Positive & 477 & $82.5 \%$ \\
\hline & Unknown & 6 & $1.0 \%$ \\
\hline \multirow[t]{3}{*}{ CD4 count ${ }^{\mathrm{a}}(n=477)$} & Low $\left(\leq 100\right.$ cells $\left./ \mathrm{mm}^{3}\right)$ & 173 & $36.3 \%$ \\
\hline & $>100$ cells $/ \mathrm{mm}^{3}$ & 198 & $41.5 \%$ \\
\hline & Missing & 106 & $22.2 \%$ \\
\hline \multirow[t]{8}{*}{ ART status $(n=477)$} & Not on ART & 116 & $24.3 \%$ \\
\hline & Median CD4 count & 156.5 & IQR: 65, 255 \\
\hline & $\begin{array}{l}\text { Initiated ART with or } \\
\text { after RR TB }\end{array}$ & 153 & $32.1 \%$ \\
\hline & Median CD4 count & 100.5 & IQR: 42.5, 221.5 \\
\hline & $\begin{array}{l}\text { Median days RR TB at } \\
\text { ART initiation }\end{array}$ & 26 & IQR: 14,42 \\
\hline & $\begin{array}{l}\text { On ART prior to RR TB } \\
\text { initiation }\end{array}$ & 209 & $43.8 \%$ \\
\hline & Median CD4 count & 101 & IQR: 41, 253 \\
\hline & $\begin{array}{l}\text { Median days on ART at } \\
\text { RR TB initiation }\end{array}$ & 332 & IQR: 160, 991 \\
\hline \multirow[t]{6}{*}{ ART regimen $(n=362)$} & $\mathrm{TDF}+3 \mathrm{TC}$ or $\mathrm{ETC}+\mathrm{EFV}$ & 182 & $50.23 \%$ \\
\hline & $\mathrm{D} 4 \mathrm{~T}$ or $\mathrm{AZT}+3 \mathrm{TC}+\mathrm{EFV}$ & 34 & $9.4 \%$ \\
\hline & $T D F+3 T C+L P V / r$ & 3 & $0.8 \%$ \\
\hline & D4T or AZT $+3 T C+L P V / r$ & 46 & $12.7 \%$ \\
\hline & Other regimen & 53 & $14.6 \%$ \\
\hline & Missing & 44 & $12.2 \%$ \\
\hline \multirow[t]{5}{*}{ Reported co-morbidities } & $\begin{array}{l}\text { Hepatitis or liver } \\
\text { disorder }\end{array}$ & 7 & $1.2 \%$ \\
\hline & Epilepsy & 6 & $1.0 \%$ \\
\hline & Psychiatric disorder & 5 & $0.9 \%$ \\
\hline & Diabetes mellitus & 9 & $1.6 \%$ \\
\hline & Renal dysfunction & 8 & $1.4 \%$ \\
\hline Pregnancy $(n=295)$ & Pregnant & 7 & $2.4 \%$ \\
\hline Contraception ( $n=295$ ) & $\begin{array}{l}\text { Using hormonal } \\
\text { contraceptive }\end{array}$ & 16 & $5.4 \%$ \\
\hline
\end{tabular}

$A R T$ antiretroviral therapy, TDF tenofovir, 3TC lamivudine, EFV efavirenz, $L P V / r$ lopinavir/ritonavir

${ }^{\mathrm{a}} \mathrm{CD} 4$ count at RR TB treatment initiation

severe AE (grade 3+). Patients with at least one reported $\mathrm{AE}$ experienced a median of 3 AEs (IQR: 2-4) per patient.

Gastro-intestinal AEs were the most common, with 138 reports of vomiting, nausea, abdominal pain, epigastric discomfort, diarrhoea, constipation, loss of appetite, or weight loss. Although most nausea and vomiting reported ( $67.1 \%$ of 70 reports) was mild to moderate (Fig. 1), it was the second most commonly reported severe AEs (11.8 \% of all the severe AEs). The most common severe AE reported was hearing loss or ototoxicity (Fig. 2). In total, 114 reports of hearing loss were noted

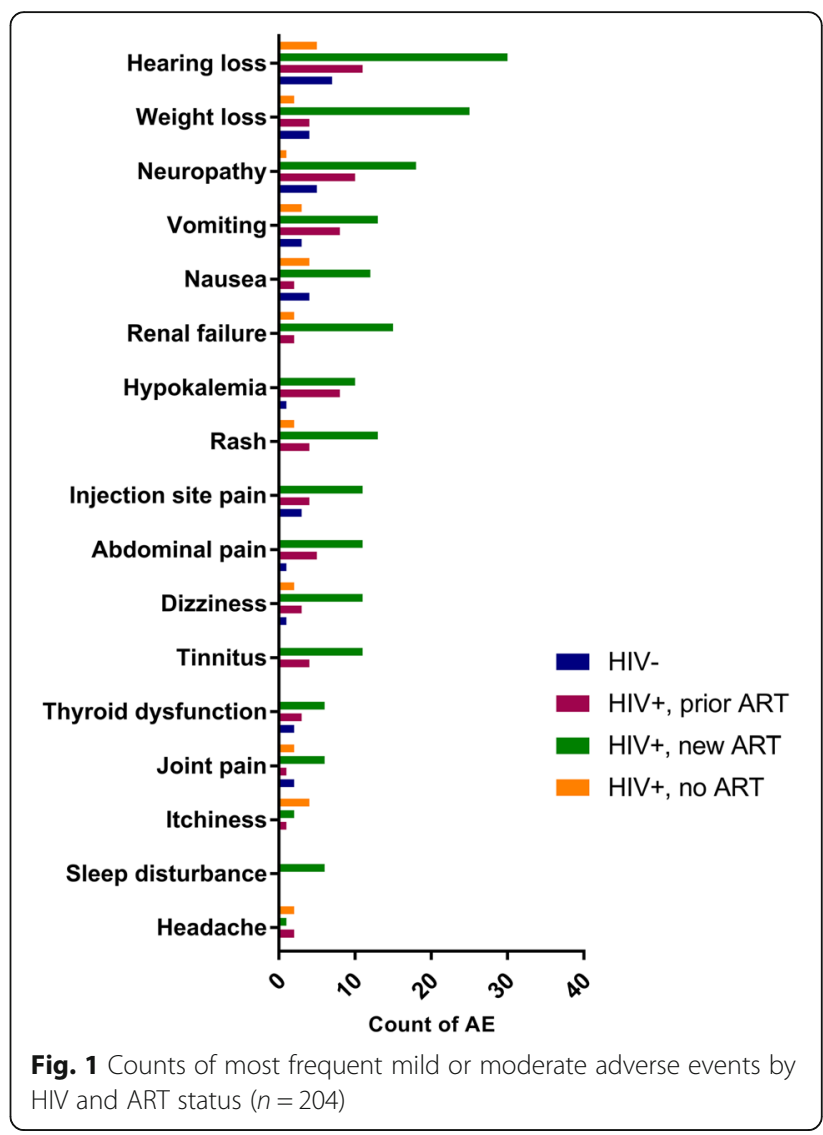

affecting $17.3 \%$ of patients $(n=100 / 578)$. Of the hearing loss AEs reported, 61 (53.5 \%) were categorised as grade $3+$. Renal dysfunction or failure accounted for $10.3 \%$ of all severe AEs, with 20 episodes reported. Psychosis (6.7 \%), neuropathy $(6.1 \%)$ and hepatitis or liver dysfunction (5.6 \%) were also among the most frequent severe AEs reported. Rare severe AE reports included deep vein thrombosis $(n=2)$, sepsis $(n=1)$, miscarriage $(n=1)$, suicidal thoughts $(n=1)$, and neuroleptic malignant syndrome $(n=1)$.

Kanamycin was listed as the suspected drug causing $\mathrm{AE}$ or severe $\mathrm{AE}$ for $54.4 \%$ (111/204) of patients experiencing an AE. Among the 309 AEs with at least one suspected drug listed, kanamycin was listed 175 times (56.6\%). Of the severe AEs with at least one suspected drug listed, terizidone was listed 32/126 times (25.4\%). Less than $5 \%(n=26)$ of the AEs had hospitalization documented as an outcome of the AE. Nearly $20 \%(n=109)$ of identified AEs resulted in the suspected drug being discontinued and an additional $10 \%(n=57)$ resulted in the dose of the suspected drug being reduced.

\section{Subdistribution hazard ratios of severe $\mathrm{AE}$}

Of the 578 patient files reviewed, 18 did not contribute to the time-to-event analysis as patient either died or were transferred out prior to returning to the clinic after 


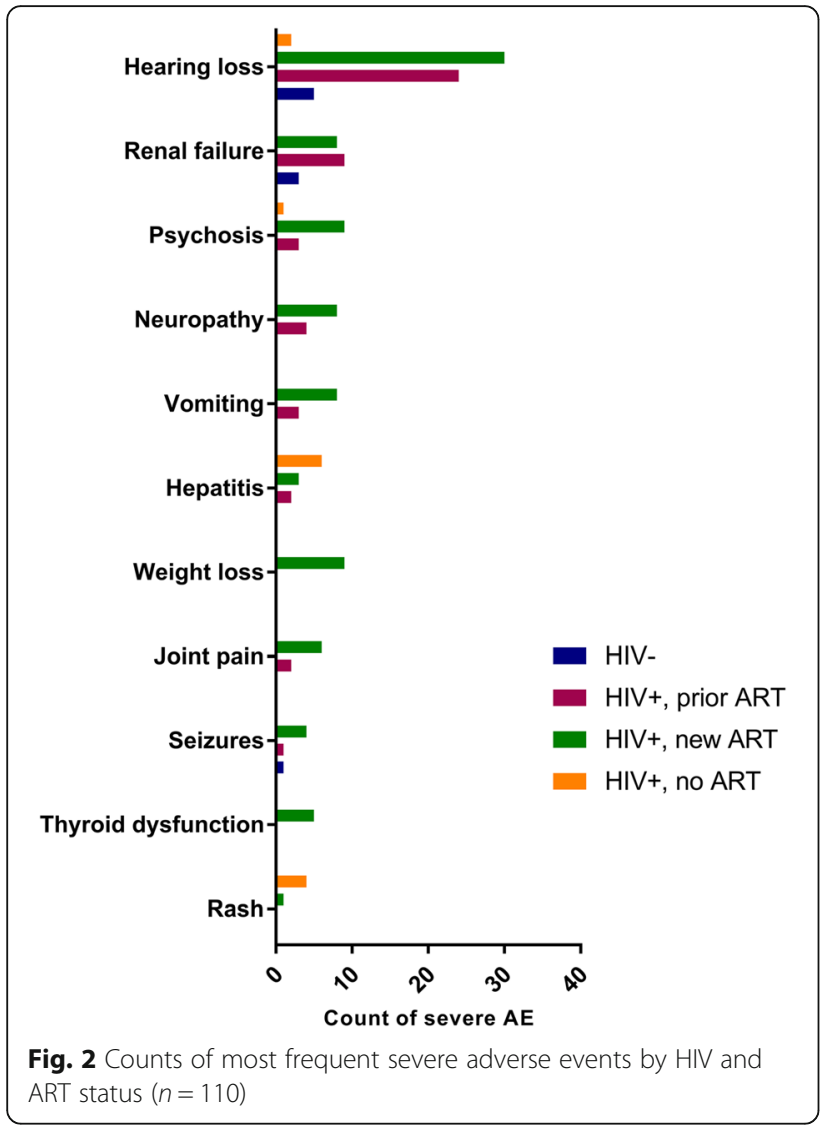

RR TB treatment initiation. The 560 that were included contributed 52,684 person-days of follow-up with a median exposure of 70 days (IQR: 28,183 ) from treatment initiation until severe AE or censoring. Of the 560, 268 (47.9\%) completed 6 months of treatment, 171 (30.5\%) were followed until transferred to another site, 73 (13.0\%) were lost from treatment, and 48 (8.6 \%) died. There were 107 severe AEs analysed, giving an incidence rate for severe $\mathrm{AE}$ of 0.74 per person year.

HIV-positive patients with low CD4 counts $(\leq 100$ cells $/ \mathrm{mm}^{3}$ ) and those who initiated ART with RR TB treatment both were approximately 3 times more likely to experience a severe AE with crude sHR: 2.76 (95\% CI: $1.30-5.84$ ) and sHR: 3.07 (95\% CI: 1.46-6.46), respectively (Table 3). Patients previously treated for TB with a regimen including streptomycin were also more likely to experience a severe AE, sHR: 3.49 (95 \% CI: 1.52-8.02), all were ototoxicity. Subdistribution hazard ratios for categorized age, low weight at treatment initiation, smear microscopy positive, recent hospitalization, sex, cough at initiation, or pre-existing renal, liver, or psychiatric conditions were not statistically different.

Competing risk regression sHR can be displayed as a graph of the cumulative incidence (of the risk analysed) function over the time at risk. Figure 3 shows patients initiating ART with or after RR TB treatment had the
Table 3 Risks of severe (grade $3+$ ) adverse events during first 6 months of RR TB treatment

\begin{tabular}{|c|c|c|c|}
\hline Characteristic & Description & $s H R^{a}$ & $95 \% \mathrm{Cl}$ \\
\hline \multirow[t]{4}{*}{ Age category } & 10-24 years & 0.70 & $0.31-1.58$ \\
\hline & $25-39$ years & Referent & \\
\hline & 40-54 years & 0.92 & $0.61-1.39$ \\
\hline & 55 years + & 1.93 & $0.85-4.37$ \\
\hline \multirow[t]{3}{*}{ HIV and CD4 status } & HIV negative & Referent & \\
\hline & $\mathrm{HIV}+, \mathrm{CD} 4>100$ cells $/ \mathrm{mm}^{3}$ & 1.81 & $0.84-3.89$ \\
\hline & $\mathrm{HIV+,CD} 4 \leq 100$ cells $/ \mathrm{mm} 3$ & 2.76 & $1.30-5.84$ \\
\hline \multirow[t]{4}{*}{ HIV and ART status } & HIV negative & Referent & \\
\hline & HIV+, initiated ART prior to RR TB & 1.77 & $0.83-3.77$ \\
\hline & HIV+, initiated ART with or after RR TB & 3.07 & $1.46-6.46$ \\
\hline & $\mathrm{HIV}+$, not on ART & 1.15 & $0.43-3.10$ \\
\hline \multirow[t]{2}{*}{ Weight (kg) } & Weight $>51$ kg & Referent & \\
\hline & Low weight $(\leq 50$ kg) & 1.43 & $0.97-2.10$ \\
\hline \multirow[t]{3}{*}{ Prior TB treatment } & No TB history reported & Referent & \\
\hline & History of first-line TB treatment & 1.33 & $0.85-2.07$ \\
\hline & $\begin{array}{l}\text { History of streptomycin for TB } \\
\text { treatment }\end{array}$ & 3.49 & $1.52-8.02$ \\
\hline \multirow[t]{2}{*}{ Referring site } & Outpatient facility & Referent & \\
\hline & Inpatient facility & 1.11 & $0.74-1.70$ \\
\hline \multirow[t]{2}{*}{ Sex } & Female & Referent & \\
\hline & Male & 0.83 & $0.57-1.22$ \\
\hline \multirow[t]{2}{*}{ Smear microscopy } & $\begin{array}{l}\text { Sputum smear negative or not } \\
\text { reported }\end{array}$ & Referent & \\
\hline & $\begin{array}{l}\text { Sputum smear positive } \\
\text { (scanty or higher) }\end{array}$ & 1.00 & $0.69-1.47$ \\
\hline \multirow{2}{*}{$\begin{array}{l}\text { Presenting } \\
\text { symptom }\end{array}$} & No cough & Referent & \\
\hline & Any cough & 1.37 & $0.90-2.08$ \\
\hline \multirow[t]{2}{*}{ Co-morbidities $^{\mathrm{b}}$} & $\begin{array}{l}\text { No reported pre-existing renal } \\
\text { insufficiency, liver or psychiatric } \\
\text { disorder }\end{array}$ & Referent & \\
\hline & $\begin{array}{l}\text { Pre-existing renal, liver, or } \\
\text { psychiatric condition }\end{array}$ & 0.47 & $0.11-1.93$ \\
\hline
\end{tabular}

Bolded values are statistically significant at $p$-value $<0.05$

$A R T$ antiretroviral therapy, $R R T B$ rifampicin resistant tuberculosis, $s H R$ subdistribution hazard ratio

${ }^{\mathrm{a}} \mathrm{sHR}$ crude analysis from competing risk regression accounting for death and loss from treatment

${ }^{\mathrm{b}}$ No pregnant women had a documented severe AE prior to censoring

highest sHR of experiencing a severe AE versus HIVnegative patients, HIV-positive patients who had been on ART prior to RR TB diagnosis, and HIV-positive patients never on ART. As a comparison, the cumulative incidence function of death during RR TB treatment, accounting for the competing risk of loss from treatment, is shown for the same HIV and ART categories (Fig. 4). HIV-positive patients who did not initiate ART either before or at RR TB initiation were more than 3 times more likely to die during RR TB treatment than HIV-negative patients (sHR: 3.25, 95 \% CI: 1.17-9.02). Figure 4 shows 


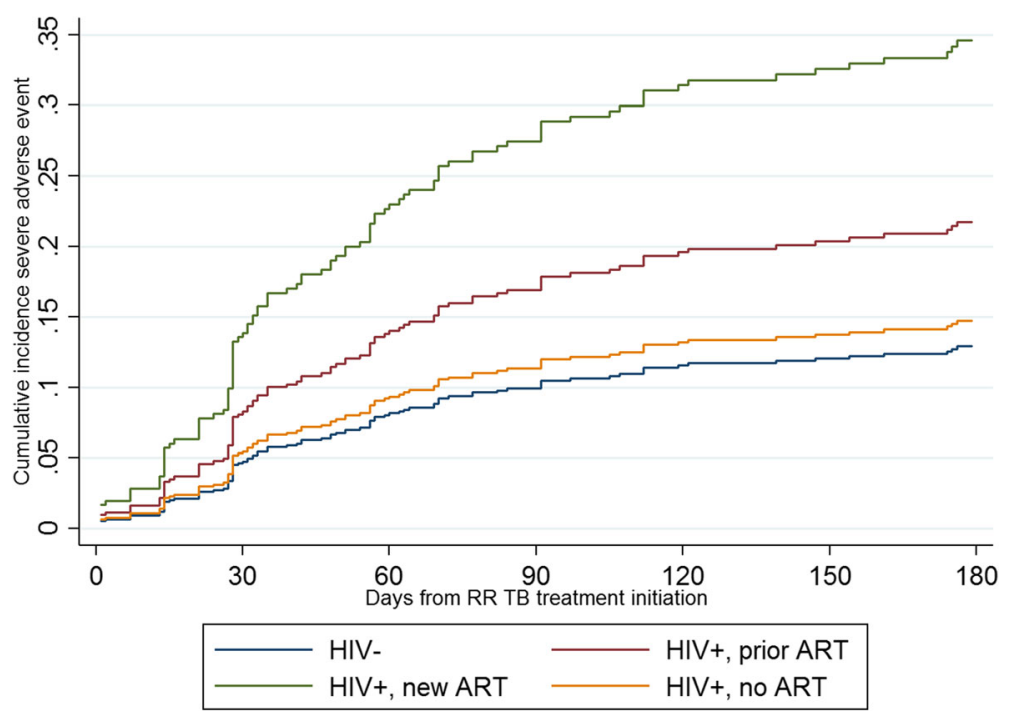

Fig. 3 Cumulative incidence function after competing risk regression of any severe adverse event. Legend: Competing risk accounting for loss from treatment and death during treatment, by HIV and ART status

the cumulative incidence function for mortality during second-line TB treatment was highest for HIV-positive patients never on ART versus HIV-negative, HIV-positive patients who had been on ART prior to RR TB diagnosis, and HIV-positive patients initiating ART along with or after RR TB treatment.

\section{Discussion}

During the first six months of RR TB treatment in a context where $82.5 \%$ of patients were also infected with HIV, $35.3 \%$ of patients experienced at least $1 \mathrm{AE}$. This incidence was less than reported in a meta-analysis of adverse drug events during the 18 to 24 months of treatment of MDR-TB, which found $57.3 \%$ of patients (260/ 534) experienced at least one $\mathrm{AE}$ [6]. One of the included studies, from a similar context in South Africa, reported that $99 \%$ of the 71 patients in that cohort experienced at least one AE [17]. The meta-analysis did not report whether the AEs were mild, moderate, severe, life threatening, or fatal [6]. In our cohort, the incidence of severe AEs during the first 6 months of treatment was $19.0 \%$, far higher than the $6.9 \%$ reported in a large cohort of HIV positive, ART naïve MDR TB patients also from South Africa [18].

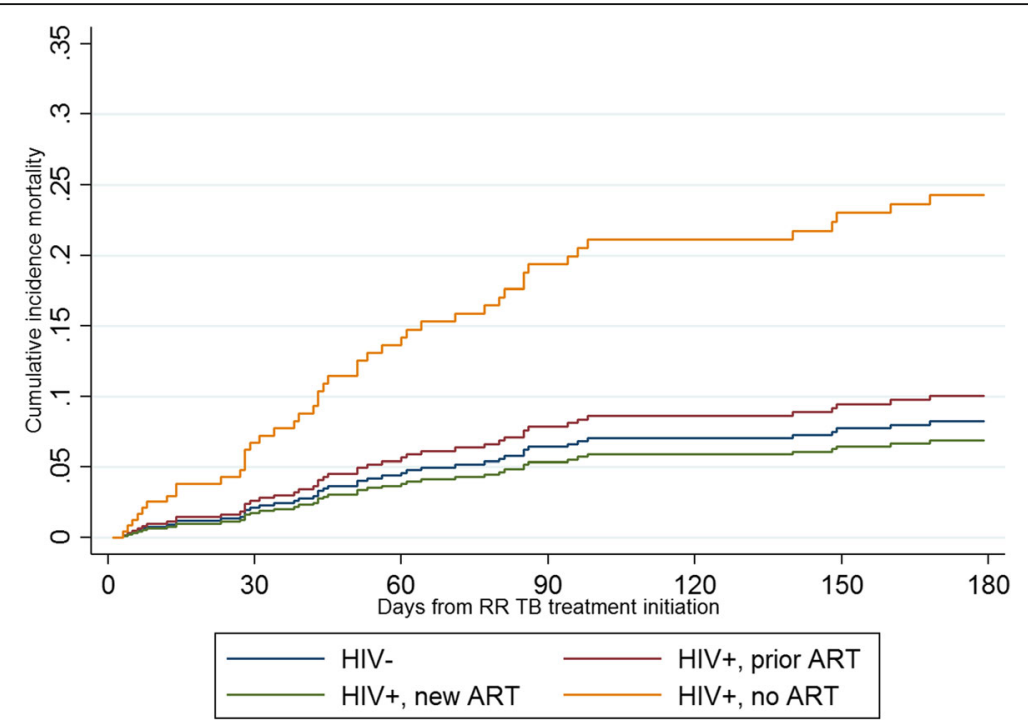

Fig. 4 Cumulative incidence function after competing risk regression of death during RR TB treatment. Legend: Competing risk accounting for loss from treatment, by HIV and ART status 
Kanamycin was listed as the suspected drug causing $\mathrm{AE}$ or severe $\mathrm{AE}$ for $54.4 \%$ of cohort patients experiencing an AE. Kanamycin, along with other aminoglycosides, is associated with ototoxicity (hearing loss and vestibular dysfunction) and renal dysfunction $[4,13,18,19]$. While an effective agent against drug-resistant TB [9], multiple trials are underway to develop an injection-free regimen for treating RR TB in an attempt to reduce the overall burden of ADR and improve adherence to long-term therapy [20].

Our finding that patients recently initiating both ART and RR TB treatment have an increased sHR for severe AE during the first 6 months (sHR: 3.07) has not been previously reported. The WHO recommendation to immediately initiate ART was based upon lower risk of death but found that there was 'very low quality of evidence' as to whether concomitant use of ART and RR TB treatment led to more severe $\mathrm{AE}$ or drug interactions [9]. A previous systematic review of the use of ART during second-line TB treatment concluded there was insufficient data as to whether concomitant use of ART and RR TB treatment increased the risk of ADR [8]. A study of XDR TB patients in South Africa found that there was no difference in the proportion of patients (using chi-squared test) with a severe $\mathrm{AE}$ reported for HIV-positive patients on ART compared to HIV-negative patients [21]. Our finding of the higher sHR for severe AE may be because other studies did not differentiate the grade of $\mathrm{AE}$, had insufficient numbers of patients on ART, or did not differentiate the time on ART. Additionally, using the competing risk methodology to calculate sHR accounting for the competing risk of death and loss from treatment was useful in the context of very high early mortality for patients not on ART. The increased sHR point to a need for additional or more frequent monitoring for $\mathrm{AE}$ for patients initiating both ART and RR TB treatment at the same time. These patients may also benefit from inpatient treatment initiation where they can be more closely monitored and managed.

The cumulative incidence function for mortality accounting for the competing risk of loss to treatment indicated that patients not on ART are most at risk of death and therefore our results are consistent with studies and guidelines that indicate early initiation of ART for patients with TB [22-24] and drug-resistant TB $[8,9]$. This reversal of risk (patients with highest relative SHR of mortality have the lowest relative sHR of severe $\mathrm{AE}$ ) also highlights the need for greater access to ART and initiation at higher CD4 counts. In our study, there was a high proportion of patients with very low CD4 counts not on ART despite increasing access to ARVs in South Africa; patients with CD4 $\leq 100$ cells $/ \mathrm{mm}^{3}$ also had a higher sHR of severe AE (sHR: 2.76). Further, a quarter of the patient files reviewed in this study were diagnosed with RR TB while hospitalized although testing for RIF resistance is available at the lowest levels of the public health system in South Africa. Again, this represents delays in diagnosis and case finding at the primary health care level leading to hospitalization and late diagnoses.

While most nausea and vomiting were reported as mild to moderate (67.1\% of 70 reports) this $\mathrm{AE}$ can impact on both adherence and effectiveness of treatment. Because of AEs, the treating clinicians discontinued or reduced the dosage of the suspected drug for $17.3 \%$ of patients $(100 / 578)$, a similar proportion to the $21 \% \mathrm{cal}-$ culated from a patient-level meta-analysis of MDR-TB studies [25]. Because of overlapping toxicities, a patient who experienced an AE during RR TB treatment may no longer be eligible for standard ART or second-line TB treatment regimens, for example because of druginduced liver or kidney injuries. Having fewer effective drugs in the second-line TB regimen is associated with lower probability of treatment success [25] and consequently higher risk of acquired additional resistance.

Prior exposure to streptomycin may be a risk factor for ototoxicity during RR TB treatment with increased aminoglycoside cumulative dose [13]. In our cohort, although the numbers of patients with prior exposure were small $(1.2 \%)$, this was the strongest predictor of a severe AE (sHR: 3.30), with all severe AEs related to ototoxicity. With the introduction of Xpert MTB/RIF diagnosis, the 'retreatment' regimen containing streptomycin was phased out and as of end 2013 is no longer in use in the South African NTP [26]. While the lack of history of prior TB treatment may reduce the risk of some types of ADR, from a public health perspective the $76.0 \%$ of patients in this cohort without any prior TB history is concerning as it indicates primary transmission of RR TB.

\section{Limitations}

The lower rates of ADR in this cohort may be a result of both the lack of routine reporting and retrospective study design. During the monthly visits to the outpatient clinic, patients are not prompted to self-report AE. In some cases, patients may have discussed $\mathrm{AE}$ with the counsellor, nurse, or clinician but if the healthcare worker did not note the discussion in the medical file it would not have been counted in this review. This may have resulted in an under-reporting of AEs, particularly those that were mild to moderate (grade 1-2). Conversely, clinicians may have spent more time monitoring or documenting AEs for patients who initiated treatment in poor clinical condition (e.g. hospitalized or concurrently starting on ART) compared to patients who were clinically stable.

Also, while guidelines indicate monitoring for certain AEs, implementation may differ from intended practice. One challenge that both sites noted during out study was that it was difficult to access audiology screening for 
patients due to concerns about infection control as audiology services were not co-located within the TB clinic. Additionally, our study focused on the first 6 months of treatment, the intensive phase, which includes kanamycin. It is possible that some patients who did not experience an ADR during the first 6 months would be affected in subsequent months or that an ADR would be detected in subsequent months, specifically aminoglycoside ototoxicity is known to occur even after treatment discontinuation [27].

Another limitation of this study was the inability to distinguish between the $\mathrm{AE}$ and ADR. For this reason, events described in this analysis were described as $\mathrm{AE}$ rather than the more specific term ADR. This limitation is common to studies describing ADR and AE and must be taken into consideration when trying to draw conclusions across studies. For example, in the South African study indicated above with $99 \%$ incidence of $\mathrm{AE}$, injection site pain was the second most commonly reported clinical AE. Injection site pain was only reported in this cohort for patients refusing to continue with the injections for reason of pain. Deaths were only included in the analysis if the clinician indicated that the death was likely due to an ADR; it is possible that deaths from ADR are underestimated as a result. Cause of death, particularly for outpatient care, are often not available as the patients die at home or in a different facility. This study also did not try to distinguish whether the AE was related to the ART or second-line TB treatment.

Finally, the study period preceded the roll-out of bedaquiline and linezolid in the South African NTP. These two drugs are now available for patients who experience toxicity to one of the drugs of the standard regimen [28] and use of the new and re-purposed drugs may affect the profile of ADR experienced by patients with drugresistant TB in South Africa.

\section{Conclusions}

Severe adverse events are common during the first 6 months of second-line treatment and HIV-positive patients newly initiating ART have the highest relative subdistribution hazard ratio of experiencing a severe AE, accounting for the competing risk of death and loss from treatment. Patients whose clinical condition requires immediate and concomitant initiation of ART and RR TB treatment may benefit from new RR TB treatment regimens that are better tolerated, intensive monitoring, or even inpatient treatment initially to watch for severe AEs.

\footnotetext{
Acknowledgements

The authors wish to thank the patients and staff at both facilities. A special thank you to Erika Mohr, Portia Baloyi, and Dudu Qwabe for their efforts.
}

\section{Funding}

$\mathrm{KS}, \mathrm{RB}, \mathrm{CF}$ were supported by a cooperative agreement from the US Agency for International Development (USAID) to Right to Care \#674-A-12-00020. AB,
NM were supported by USAID agreement number \#674-12-00002 to Wits Reproductive Health and HIV Research Institute. DE was supported through USAID agreement number \#674-A-12-00029 to the Health Economics and Epidemiology Research Office. The contents of the article are the responsibility of the authors and do not necessarily reflect the views of USAID or the US government. The funders had no role in the study design, collection, analysis and interpretation of the data, in manuscript preparation or the decision to publish. NM is an employee of GlaxoSmithKline (GSK); however, the study was conducted prior to her employment with GSK. GSK has not sponsored or funded the study in any way. The contents are the responsibility of the authors and not of GSK.

Availability of data and materials

The dataset(s) supporting the conclusions of this article is (are) included within the article (and its additional file(s)).

\section{Authors' contributions}

$\mathrm{KS}, \mathrm{RB}$ conceived of the study. $\mathrm{RB}, \mathrm{AB}, \mathrm{NM}$ reviewed patient files and extracted data. KS designed and performed statistical analysis and wrote manuscript. RB, CF, AB, ES, DE reviewed analysis and edited manuscript. All authors read and approved the final manuscript.

\section{Authors' information}

Not applicable.

\section{Competing interests}

The authors declare that they have no competing interests.

\section{Consent for publication}

Not applicable.

\section{Ethics approval and consent to participate}

Ethical approval without patient informed consent for this de-identified retrospective medical record review was obtained from the Human Research Ethics Committees of the University of the Witwatersrand and the University of Cape Town.

\section{Author details}

${ }^{1}$ Right to Care, Johannesburg, South Africa. ${ }^{2}$ Clinical HIV Research Unit, School of Clinical Medicine, Faculty of Health Sciences, University of the Witwatersrand, Johannesburg, South Africa. ${ }^{3}$ Health Economics Unit, School of Family and Public Health, Faculty of Health Sciences, University of Cape Town, Cape Town, South Africa. ${ }^{4}$ Health Economics and Epidemiology Research Office, Department of Internal Medicine, School of Clinical Medicine, University of the Witwatersrand, Johannesburg, South Africa. ${ }^{5}$ School of Medicine, University of North Carolina, Chapel Hill, USA. ${ }^{6}$ Wits Reproductive Health and HIV Research Institute, School of Clinical Medicine, Faculty of Health Sciences, University of the Witwatersrand, Johannesburg, South Africa. ${ }^{7}$ GlaxoSmithKline, Bryanston, South Africa.

Received: 13 February 2016 Accepted: 15 October 2016

Published online: 21 October 2016

\section{References}

1. World Health Organization (WHO). Global tuberculosis report. Geneva: World Health Organization; 2015.

2. Statistics South Africa. Mortality and causes of death in South Africa, 2014: Findings from death notification. Pretoria: Statistics South Africa; 2015. www. statssa.gov.za. doi: Statistical release P0309.3.

3. World Health Organization (WHO). Definitions and reporting framework for tuberculosis-2013 revision. 2013.

4. Directorate Drug-Resistant TB. Management of drug-resistant TB: policy guidelines. Pretoria: National Department of Health; 2013. doi:10.5772/56396.

5. World Health Organization (WHO). A practical handbook on the pharmacovigilance of medicines used in the treatment of tuberculosis: enhancing the safety of the TB patient. 2012.

6. Wu S, Zhang Y, Sun F, Chen M, Zhou L, Wang N, et al. Adverse events associated with the treatment of multidrug-resistant tuberculosis: a systematic review and meta-analysis. Am J Ther. 2013. doi:10.1097/01.mjt. $0000433951.09030 .5 a$. 
7. Schnippel K, Shearer K, Evans D, Berhanu R, Dlamini S, Ndjeka N. Predictors of mortality and treatment success during treatment for rifampicin-resistant tuberculosis within the South African National TB Programme, 2009 to 2011: a cohort analysis of the national case register. Int J Infect Dis. 2015;39:89-94. doi:10.1016/j.jijid.2015.09.002.

8. Arentz M, Pavlinac P, Kimerling ME, Horne DJ, Falzon D, Schünemann HJ, et al. Use of anti-retroviral therapy in tuberculosis patients on second-line anti-TB regimens: a systematic review. PLoS One. 2012;7:3-12. doi:10.1371/ journal.pone.0047370.

9. World Health Organization (WHO). Guidelines for the programmatic management of drug-resistant tuberculosis. 2011 updat. Geneva: World Health Organization; 2011.

10. Directorate Drug-Resistant TB, DR-TB Directorate. Introduction of new drugs and drug regimens for the management of drug-resistant tuberculosis in south africa: policy framework. 11th ed. Pretoria: National Department of Health; 2015.

11. South African National Department of Health. Clinical guidelines for the management of HIV and AIDS in adults and adolescents. Pretoria: South African National Department of Health; 2010. Document available from http://apps.who.int/medicinedocs/documents/s19153en/s19153en.pdf.

12. South African National Department of Health. The South African Antiretroviral Treatment Guidelines. Pretoria: South African National Department of Health; 2013. Document available from http://www.sahivsoc.org/Files/2013\%20ART\%20Treatment \%20Guidelines\%20Final\%2025\%20March\%202013\%20corrected.pdf.

13. Seddon J a, Godfrey-Faussett P, Jacobs K, Ebrahim A, Hesseling AC, Schaaf HS. Hearing loss in patients on treatment for drug-resistant tuberculosis. Eur Respir J. 2012;40:1277-86. doi:10.1183/09031936.00044812.

14. Mehta U, Dheda M, Steel G, Blockman M, Ntilivamunda a, Maartens G, et al. Strengthening pharmacovigilance in South Africa. S Afr Med J. 2014;104: 104-6. doi:10.7196/SAMJ.7517.

15. AIDS Clinical Trials Group. Division of AIDS table for grading the severity of adult and pediatric adverse events. 2009. p. 1-21.

16. Fine JP, Gray RJ. A Proportional hazards model for the subdistribution of a competing risk. J Am Stat Assoc. 2009;94:496-509.

17. Brust JCM, Shah NS, van der Merwe TL, Bamber S, Ning Y, Heo M, et al. Adverse events in an integrated home-based treatment program for MDRTB and HIV in KwaZulu-Natal, South Africa. J Acquir Immune Defic Syndr. 2013:62:436-40. doi:10.1097/QAl.0b013e31828175ed.

18. Van der Walt M, Lancaster J, Odendaal R, Davis JG, Shean K, Farley JE. Serious treatment related adverse drug reactions amongst anti-retroviral naïve MDR-TB patients. PLoS One. 2013;8:e58817. doi:10.1371/journal.pone.0058817.

19. Mathews A, Bailie GR. Clinical pharmacokinetics, toxicity and cost effectiveness analysis of aminoglycosides and aminoglycoside dosing services. J Clin Pharm Ther. 1987;12:273-91.

20. Clayden P, Collins S, Daniels C, Frick M, Harrington M, Horn T, et al. TAG 2015 pipeline report. London: HIV i-Base/Treatment Action Group; 2015.

21. Shean K, Streicher E, Pieterson E, Symons G, van Zyl Smit R, Theron G, et al. Drug-associated adverse events and their relationship with outcomes in patients receiving treatment for extensively drug-resistant tuberculosis in South Africa. PLoS One. 2013;8:e63057. doi:10.1371/journal.pone.0063057.

22. Havlir DV, Kendall M a, Ive P, Kumwenda J, Swindells S, Qasba SS, et al. Timing of antiretroviral therapy for HIV-1 infection and tuberculosis. N Engl J Med. 2011;365:1482-91. doi:10.1056/NEJMoa1013607.

23. Blanc F-X, Sok T, Laureillard D, Borand L, Rekacewicz C, Nerrienet E, et al. Earlier versus later start of antiretroviral therapy in HIV-infected adults with tuberculosis. N Engl J Med. 2011;365:2187-98. doi:10.1056/NEJMoa1011205.

24. Abdool Karim SS, Naidoo K, Grobler A, Padayatchi N, Baxter C, Gray A, et al. Timing of initiation of antiretoviral drugs during tuberculosis therapy. $\mathrm{N}$ Engl J Med. 2010;362:697-706. doi:10.1056/NEJMoa0905848.

25. Ahuja SD, Ashkin D, Avendano M, Banerjee R, Bauer M, Bayona JN, et al. Multidrug resistant pulmonary tuberculosis treatment regimens and patient outcomes: an individual patient data meta-analysis of 9,153 patients. PLoS Med. 2012;9:e1001300. doi:10.1371/journal.pmed.1001300.

26. TB DOTS Strategy Coordination. National tuberculosis management guidelines 2014. Pretoria: National Department of Health; 2014. http://www.sahivsoc.org/ Files/NTCP_Adult_TB\%20Guidelines\%2027.5.2014.pdf.

27. Selimoglu E. Aminoglycoside-induced ototoxicity. Curr Pharm Des. 2007;13: 119-26. doi:10.2174/138161207779313731.

28. Directorate Drug-Resistant TB. Introduction of new drugs, drug regimens and management for drug-resistant TB in South Africa: policy framework. 11th ed. Pretoria: National Department of Health; 2015.

\section{Submit your next manuscript to BioMed Central and we will help you at every step:}

- We accept pre-submission inquiries

- Our selector tool helps you to find the most relevant journal

- We provide round the clock customer support

- Convenient online submission

- Thorough peer review

- Inclusion in PubMed and all major indexing services

- Maximum visibility for your research

Submit your manuscript at www.biomedcentral.com/submit 